ORIGINAL ARTICLE

\title{
Psychosocial work factors and dementia
}

\author{
A Seidler, A Nienhaus, T Bernhardt, T Kauppinen, A-L Elo, L Frölich
}

Occup Environ Med 2004;61:962-971. doi: 10.1136/oem.2003.012153

See end of article for authors' affiliations

.....................

Correspondence to:

Dr A Seidler, MPH, Institut

für Arbeitsmedizin, Johann

Wolfgang Goethe-

Universität, Theodor-Stern-

Kai 7, D-60590 Frankfurt/

Main, Germany;

a.seidler@

em.uni-frankfurt.de

Accepted 27 August 2004

\begin{abstract}
Aims: To evaluate the association between psychosocial workplace factors and dementia in a case-control study.

Methods: Patients with dementia (aged 55-99 years) were recruited from 23 general practices in the city of Frankfurt-on-Main and surrounding area in 1998-2000. Of these, 108 were suffering from possible Alzheimer's disease, 59 from possible vascular dementia, and 28 from secondary or unclassified dementia. A total of 229 control subjects (aged 60-94 years) was recruited from the same study region: 122 population controls and 107 dementia-free ambulatory patients. A detailed job history was elicited in a structured personal interview (next-of-kin interviews of cases). Psychosocial work exposure was assigned to cases and control subjects by linking lifetime job histories with a Finnish job-exposure matrix. Data were analysed using logistic regression, to control for age, region, sex, dementia in parents, education, smoking, and the psychosocial network at age 30.

Results: There were decreased odds ratios for high challenge at work, high control possibilities at work, and high social demands at work. High risks for error at work revealed a significant positive association with the diagnosis of dementia. Restriction of the analysis to cases with possible Alzheimer's disease or to cases with possible vascular dementia led to similar results.

Conclusions: These results support a role for psychosocial work factors in the aetiology of dementia. As an alternative explanation, people might have chosen jobs with poor work factors due to preclinical dementia, which becomes clinically manifest decades later.
\end{abstract}

$\mathrm{R}$ esults from several recent studies point to an association between dementia and psychosocial factors: a positive alone, having no close social ties, ${ }^{1}$ scarcely participating in social and leisure activities, ${ }^{2-4}$ and never having married. ${ }^{5}$ Recent studies have found that Alzheimer's disease in particular is negatively associated with diversity of activities and intensity of intellectual activities, ${ }^{67}$ and positively associated with psychosocial inactivity, ${ }^{8}$ unproductive working style, living with a dominant spouse," and with physical inactivity. ${ }^{8}{ }^{10}$ The results of an own case-control study ${ }^{11}$ point to a possible independent protection against dementia from social relationships, and from physical and intellectual activities in midlife, possibly also in later life. In this case-control study, adjustment for the psychosocial network neutralises the otherwise protective effect of education on dementia of any type and on possible vascular dementia.

While there is growing scientific evidence for a relation between dementia and psychosocial factors in leisure time, up to now no studies have dealt with the potential aetiological relevance of psychosocial working conditions. Our case-control study is the first epidemiological study to analyse psychosocial work factors as risk factors for dementia.

Dementia patients are not usually able to report previous exposures accurately. In case-control studies of dementia, the data collection-at least in cases-therefore has to be based on next-of-kin interviews. As the assessment of the psychosocial work factors by the next-of-kin of cases could lead to substantial recall bias, we decided to base the assessment of psychosocial work factors on a job-exposure matrix constructed by Kauppinen and colleagues. ${ }^{12}$

\section{METHODS}

\section{Study population}

The study design has been described in detail in a previous publication. ${ }^{11}$ Briefly, patients with dementia as well as control subjects come from the city of Frankfurt-on-Main and from the neighbouring cities of Darmstadt, Offenbach, and Bad Homburg. Patients with dementia were recruited in 23 general practices in 1998-2000. Participating physicians were asked to review in detail their clinical records in order to identify all demented patients 65 years of age or more (incident and prevalent cases) within their clientele. If available, the manually revealed information was cross checked by computer based data files. Of 287 eligible patients, 221 agreed to participate (77\%). To substantiate the cognitive deficit, mini mental state examinations (MMSE) were applied by trained interviewers (one psychologist and three medical students). On each patient's entry in the study, one psychiatrist (LF) reviewed the medical findings, including the results of work-ups performed by neurologists and by the results of magnetic resonance imaging (MRI) or $x$ ray computed tomography (CT), if available.

The differential diagnosis between Alzheimer's disease $(\mathrm{AD})$, vascular dementia (VD), and secondary dementia was based on the German version of ICD-10. ${ }^{13}$ Only in $23.6 \%$ of the cases $(n=46)$ were MRI or CT brain scans available. However, several guidelines for the diagnosis of Alzheimer's disease and vascular dementia in clinical practice do not require a CT or MRI as an obligatory diagnostic investigation. ${ }^{14}{ }^{15}$ Following these diagnostic criteria (CT/MRI not diagnostically obligatory) the differentiation between probable Alzheimer's disease (AD) and probable vascular dementia (VD) can be achieved with a specificity of about $80 \%$. As our differentiation between Alzheimer's disease and vascular dementia is of limited diagnostic validity, we use the diagnostic classes "possible Alzheimer's disease" and "possible vascular dementia".

Patients with an MMSE score $>26(\mathrm{n}=23)$, patients suffering from depressive disorder with acute cognitive symptoms $(n=1)$, as well as patients whose first cognitive symptoms had started more than 20 years ago $(n=2)$, were 


\section{Main messages}

- Challenging work with high control possibilities and high social demands might prevent the development of dementia or at least delay the time of its clinical manifestation.

- As an alternative explanation, preclinical symptoms of dementia might influence the career decades before the dementia becomes clinically manifest.

excluded from the study. In cases of inconsistency between the CT/MRI findings, the external neurological diagnosis, and the Hachinski score, diagnosis was reached by means of a multidisciplinary consensus conference, including consultation of the responsible general practitioner or neurologist, as required. Altogether 195 cases with dementia were included in the analysis. Of these, 108 suffered from possible Alzheimer's disease (AD) (55.4\%), 59 from possible vascular dementia (VD) (30.3\%), 25 from secondary dementia diagnosis $(12.8 \%)$, and three from unclassified dementia $(1.5 \%)$.

Population control subjects were randomly selected from a $1 \%$ random sample of Frankfurt residents aged 65 years or more, drawn from the Frankfurt population registration office. To achieve an age distribution comparable to that of the cases, a stratified sample under-representing people below 80 years was drawn. Of 228 population controls, 139 agreed to participate $(61 \%)$. Potential population controls were excluded from the study if they had an MMSE score of 26 or less $(n=17)$.

As a second control group, we selected dementia-free patients in nine of the above mentioned general practices. We recruited all dementia-free patients who contacted any of these practices with any complaints on a priori defined days. Of 128 ambulatory patients, 115 agreed to participate (90\%). Eight patients had an MMSE score of 26 or less and were excluded from the study. A total of 229 control subjects was included in the analysis-122 population controls and 107 dementia-free ambulatory patients.

\section{Data collection}

Interviews were conducted by one psychologist and three medical students, who had been intensively trained in standardised interview techniques and a non-differential approach to cases and controls. The interviewers elicited information about the medical history including: dementia in parents; parental age at birth; neurological diseases of the affected person; socioeconomic factors; the psychosocial network, including marital status, living situation, social ties, and leisure activities; smoking; and alcohol consumption. The interviewers documented a complete occupational history for each participant. For every job held, information was elicited about the start and the end of the job phase, about job title, industry, and specific job tasks.

The interview was administered to the next-of-kin of the cases-mostly child $(40 \%)$ or partner $(22 \%)$-and to the control subjects themselves. When patients were suffering from the first signs of dementia and no surrogates were available $(\mathrm{n}=37 ; 19 \%$ of the cases; mean MMSE score $21.8 \pm 4.3)$, they were asked to answer the questions themselves. However, according to a preliminary analysis, exclusion of these patients did not fundamentally alter the results. In $3.1 \%$ of the control subjects $(n=7)$ the interview was administered to the next-of-kin. Twenty two control subjects did not participate in the detailed personal interview but answered a short telephone interview.

\section{Policy implications}

- To further clarify the association between specific psychosocial work factors and dementia, there is a need for long term prospective studies with prospective assessment of the psychosocial work factors.

- Further pathological knowledge about the preclinical course of dementia is needed to determine whether the association between psychosocial factors and dementia is causal.

The date of symptom onset was defined as the year of the first recognition of extraordinary forgetfulness by the nextof-kin of cases. In cases, only exposures up to the date of dementia onset as reported by the patients' next-of-kin (median 4 years) were considered for inclusion in the analysis. In control subjects, only exposures up to 4 years prior to the data collection were considered.

\section{Exposure assessment}

The psychosocial work exposure was assigned to cases and control subjects by linking lifetime job titles with an a priori job exposure matrix (JEM) constructed by Kauppinen and colleagues. $^{12}$ This matrix (so-called "FINJEM") was constructed to include the most relevant physical, chemical, microbiological, ergonomic, and psychosocial exposures or stress factors occurring in Finland. Agents, occupations, and calendar time (period) are the basic dimensions to which exposure is assessed. The occupational dimension of FINJEM is divided according to the Combined Occupational Classification of Finnish Censuses in 1970-1985, and it includes 311 categories. The FINJEM assessment of psychosocial work factors is based on the 1990 Quality of Work Life Survey. The number of items (questions) in each psychosocial factor scale varies between three and five, and the internal consistency coefficient of each psychosocial factor scale (Cronbach's alpha coefficient) is between 0.64 and 0.81 . For the following psychosocial factors the FINJEM estimates the job specific exposure level (rounded to the closest integer) on a composite scale (1-5):

- Challenge at work (based on the 1990 Quality of Work Life Survey responses to four questions concerning variability, meaningfulness, and interesting tasks at work)

- Social climate at work (based on four questions concerning the degree of open communication, information flow, and cooperation)

- Control possibilities at work (based on five questions concerning the possibilities for independence and to influence one's working pace and methods)

- Work load (based on four questions concerning the demand to work under tight schedules and time pressure, and to adjust conflicting demands from others)

- Perceived risks for error at work (based on three questions concerning the risk to meet with or cause an accident or damage property due to an error in the normal course of action)

- Social demands at work (based on three questions concerning the demand to communicate with people other than workmates, to serve and understand other people)

- Supervisor support (based on five questions concerning encouragement, trust, and support from the supervisor, communications with the supervisor). 
The FINJEM assessment of working time arrangements (on a scale $0-2$ ) is based on the Quality and Working Life Survey responses to one question concerning the exposure to different working time arrangements, with emphasis on night work.

To apply the FINJEM in our study, coding of the occupations was based on the Occupational Classification of Finnish Censuses. All jobs (on average 3.3 jobs in male dementia patients, 2.4 jobs in female dementia patients; 3.9 jobs in male control subjects, 3.0 jobs in female control subjects) were included into the calculation of a time weighted mean score of the psychosocial work load. For every job held, the FINJEM score of each psychosocial work factor (on an integer scale from 1 to 5 ; for working time arrangements on an integer scale from 0 to 2 ) was multiplied by the corresponding duration of the job phase and summed up. To calculate time weighted mean scores of psychosocial work factors, the sum was divided by the number of working years. The resulting mean scores were categorised as follows: "low to moderate" (reference category) if the mean score was 3.5 or less; "high" if the mean score was between 3.5 and 4.5; and "very high" if the mean score was 4.5 or more. If, for a single psychosocial workload factor, half of the control subjects had a mean score below 3, the categories were formed as follows: "low" (reference category) if the mean score was 2 or less; "moderate" if the mean score was between 2 and 3; and "high" if the mean score was 3 or more. The FINJEM classifies the probability (proportion of exposed workers) as well as the intensity of stressful working time arrangements (shift or night work). For each occupation, the intensity of stressful working time arrangements was weighted by probability of exposure according to the FINJEM. The time weighted mean values of the resulting products were categorised as follows: regular daytime work (reference category, mean score equal 0); little shift or night work (mean score greater than 0 , smaller than 0.5 ); moderate to high exposure to shift or night work (mean score 0.5 or more).

\section{Agreement between subjects and next-of-kin}

In our study, the assessment of psychosocial workplace exposure was based exclusively on the reported job titles. Nevertheless, differences between subjects and next-of-kin in the recall of job titles and duration of job phases could have introduced bias in the assessment of psychosocial work load. To evaluate the agreement between the subjects and next-ofkin on psychosocial work variables, in 49 interviewed control subjects, next-of-kin were also interviewed. We calculated kappa $(\kappa)$ values to compare the categorised psychosocial workload factors derived from the control subject's job declarations with the corresponding factors derived from the next-of-kin interview (see table 1). Furthermore, we used the Wilcoxon test to compare the interval scaled mean scores of the psychosocial work factors derived from the self reported job titles with the corresponding mean scores based on the next-of-kin-reported job titles. Kappa values were greater than 0.60 for all single psychosocial workload factors except for the "work load" $(\kappa=0.48)$. However, the median of the interval scaled scores for the "work load" variable did not significantly differ between subjects and next-of-kin (Wilcoxon test: $\mathrm{p}=0.67$ ). Applying the Wilcoxon test, we did not find any significant differences. However, subjects tended to report job histories that were connected with a slightly higher exposure to "different working time arrangements" in the FINJEM matrix than next-of-kin $(p=0.08)$. Altogether, there is no evidence for a systematic over- or underestimation of psychosocial work load on the basis of the next-of-kin job history. We nevertheless cannot totally exclude that the presence of a demented subject in the family might have influenced the next-of-kin report of the job titles. However, we believe that "bare facts" as job titles might to a low extent be influenced by the knowledge about the dementia status.

\section{Characteristics of cases and control subjects}

Table 2 presents the characteristics of the cases with dementia and control subjects. The mean age at symptom onset of cases with any dementia $(\mathrm{n}=195)$ was $79.5 \pm 8.4$ years; of cases having $\mathrm{AD}(\mathrm{n}=108), 80.9 \pm 8.4$ years; of cases having VD $(n=59), 78.0 \pm 8.0$ years; and of cases with secondary $(\mathrm{n}=25)$ or unclassified $(\mathrm{n}=3)$ dementia, $77.1 \pm 8.7$ years. The mean age of population controls $(\mathrm{n}=122)$ four years prior to data collection (reference date) was $78.1 \pm 6.7$ years; and of ambulatory controls $(n=107)$, $72.3 \pm 7.3$ years. Of the 195 cases, $23 \%$ were male and $77 \%$ were female. Of the 229 control subjects, $33 \%$ were male and $67 \%$ were female. About $40 \%$ of the dementia patients, but only $2 \%$ of the population controls and $8 \%$ of the ambulatory dementia-free controls were living at a retirement home. In $32 \%$ of the dementia cases $(n=62)$, the dementia was classified as severe (MMSE score $<10)$. The median time interval between the end of the last job phase and the dementia diagnosis was 17 years in men, and 24 years in women. In control subjects, the median time interval between the end of the last job phase and the reference date (four years prior to the data collection) was 10 years in men, and 21 years in women.

\section{Data analysis}

Odds ratios (OR) and 95\% confidence intervals (CI) were calculated using logistic regression analysis. As age is known to be strongly associated with the occurrence of dementia, we decided to adjust for age. Age was entered into the logistic regression model in five-year categories. The residential area of the subjects (referred to as "region" in this text) was considered to be a potential confounder, because occupational exposures were suspected to differ between regions. We controlled for the psychosocial network at age 30 as a potential confounder because it was strongly associated with dementia. ${ }^{11}$ All statistical analyses were adjusted for age, region, sex, dementia in parents, education (categorised into elementary level/no graduation, secondary school level, and high school graduation), smoking (in pack-years), and the psychosocial network at age 30. All covariates were included as categorised covariates in the multivariate analyses; the categories are shown in table 2.

The psychosocial network variable contains psychosocial ties (marital status, number of confidants) and social activities (number of sports activities and cultural activities per month). Psychosocial work variables were included as categorised variables in the logistic regression model. The psychosocial network variable was categorised as follows:

0. (Reference category): poor psychosocial ties (being widowed or living alone or having $<3$ confidants) and poor social activities (no sports activities and no cultural activities)

1. Moderate psychosocial ties and/or moderate social activities (i.e., not fitting to categories 0, 2 and 3)

2. Either high psychosocial ties (living with $>3$ persons or having $>10$ confidants) or high social activities (sports activities or $>3$ cultural activities per month)

3. High psychosocial ties and high social activities. For more detailed information about the agreement between the subjects and next-of-kin on psychosocial variables, see Seidler and colleagues. ${ }^{11}$

To calculate tests for trend, the exposure scores were included as continuous-not as categorised-variables in the logistic 


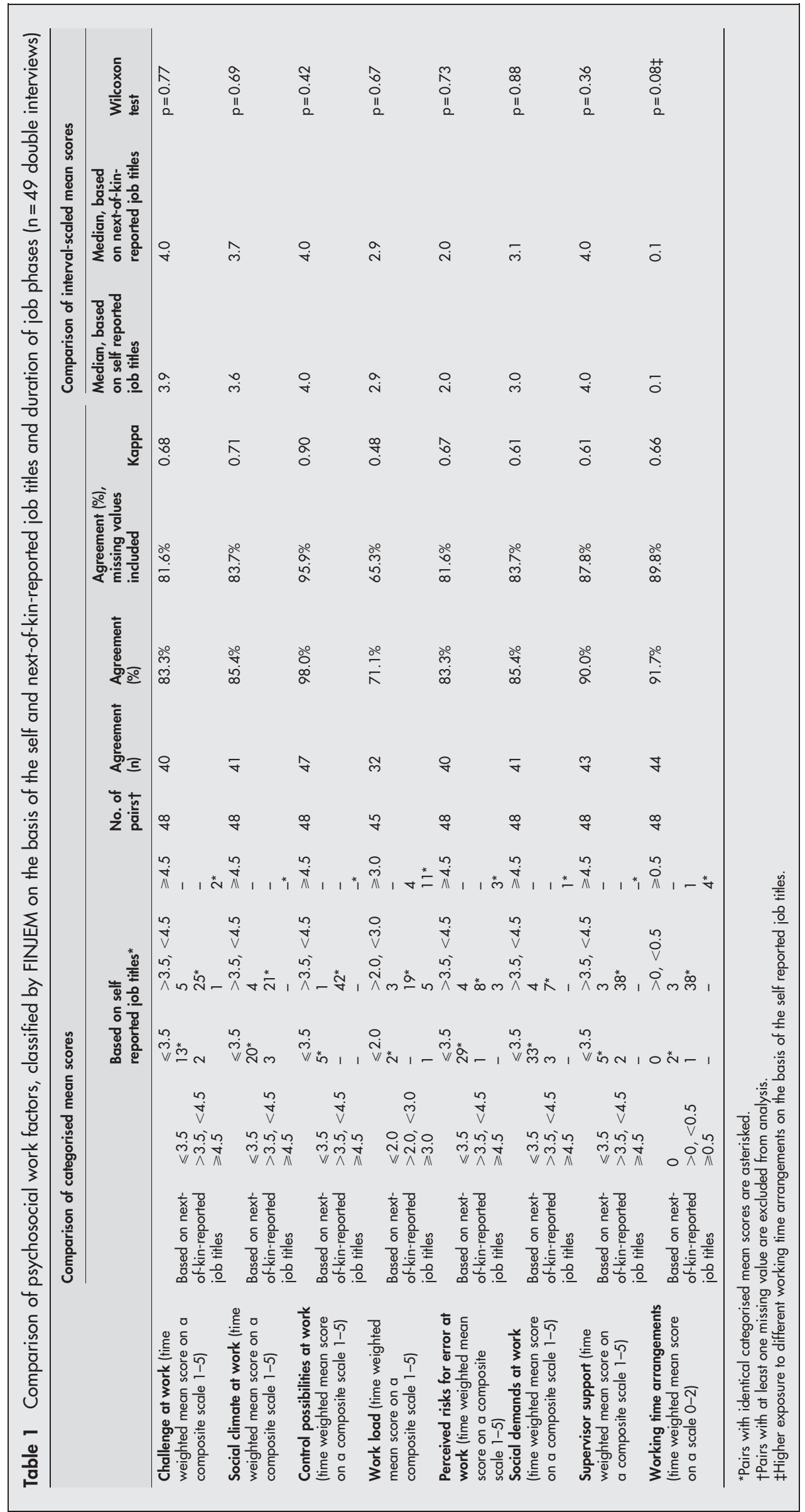




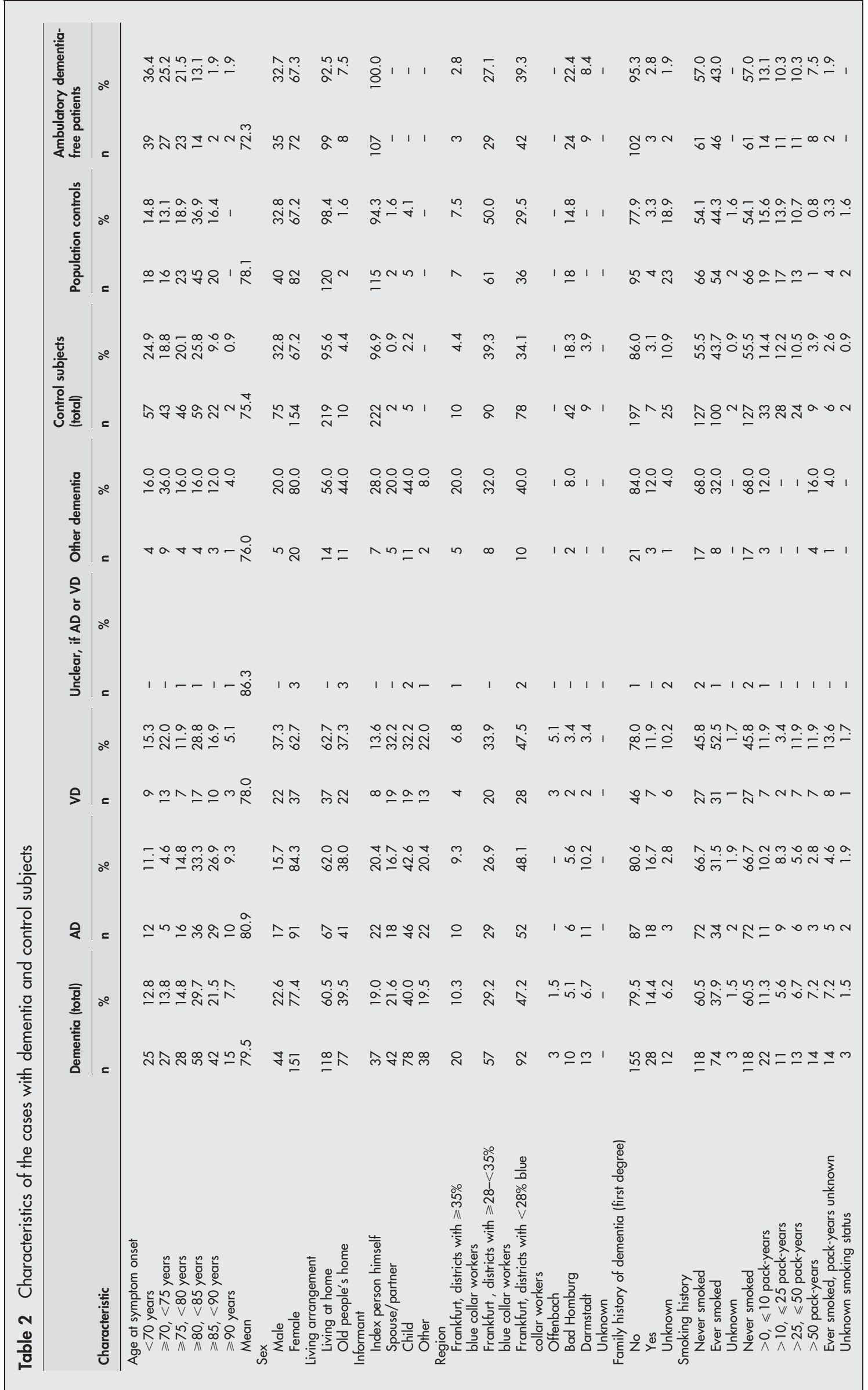




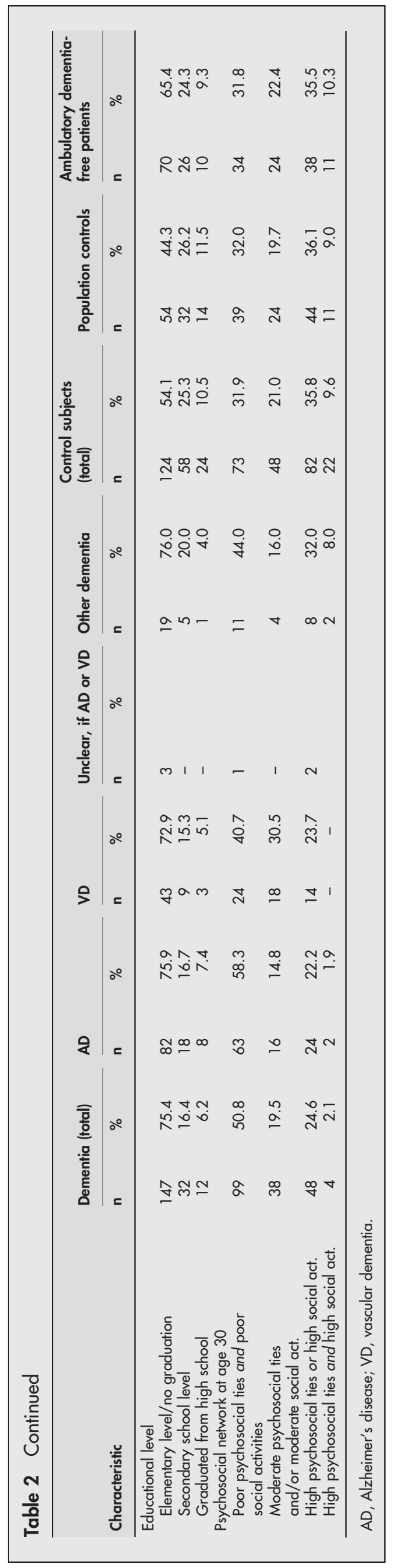

regression model. Therefore if persons fall into the same exposure category, this does not mean that their (interval scaled) mean score is identical. Subjects without any work activities as well as missing values were analysed as separate categories (results not shown here).

Firstly, we calculated the odds ratios for the single workplace factors separating between population controls and ambulatory control subjects. Secondly, we calculated the odds ratios for the single psychosocial workplace factors separately for all cases with dementia, for cases solely with possible Alzheimer's disease, and for cases solely with possible vascular dementia. Thirdly, we restricted the analysis to dementia cases without any of the following cardiovascular diagnoses: myocardial infarction; angina pectoris; stroke; transient ischaemic attacks; peripheral arterial occlusive disease; hypertension.

\section{RESULTS}

Table 3 shows the odds ratios for the association between the psychosocial workplace factors and dementia of any type, separating between the two control groups. The results of the data analysis including only population controls (table 3, left column) were comparable with the results including only ambulatory dementia-free control subjects (table 3, right column), with two exceptions. Firstly, the odds ratios (OR) for perceived risks for error at work were markedly higher for the comparison between all cases with dementia and ambulatory controls than for the comparison between cases and population controls. Secondly, restriction of the analysis to population controls led to a statistically significant association between high work load and dementia. Because of the rather good agreement between the two control groups, we combined both control groups in the final analysis to increase the power of the study.

Table 4 shows the odds ratios for the association between the psychosocial workplace factors and dementia of any type, for cases solely with possible Alzheimer's disease, and for cases solely with possible vascular dementia. Patients with dementia of any type were less likely to have experienced challenging working conditions: the relative risk as assessed by the odds ratio (OR) of having a dementia of any type (table 4, left column) was 0.5 (95\% CI 0.3 to 0.9 ) for subjects with high challenge at work (time weighted mean score of $>3.5$ to $<4.5)$ compared to subjects with low to moderate challenge at work (mean score of 3.5 or less). Two control subjects, but no dementia cases were classified as having had very high challenge at work (mean score of 4.5 or more). When only cases with possible Alzheimer's disease were included in the analysis (table 4, middle column), the odds ratio for high (versus low to moderate) challenge at work decreased to 0.3 (95\% CI 0.2 to 0.7 ); the OR was 0.5 (95\% CI 0.2 to 1.1 ) when the analysis was restricted to cases with vascular dementia (table 4, right column).

Control possibilities at work were significantly lower in dementia cases of any type than in control subjects ( $p$ for trend $=0.02)$; the OR for high control possibilities at work (time weighted mean score of $>3.5$ to $<4.5$ ) versus low to moderate control possibilities at work (time weighted mean score 3.5 or less) was 0.5 ( $95 \%$ CI 0.3 to 1.1 ). When only cases with possible vascular dementia were included in the analysis, the negative association with control possibilities remained statistically significant ( $\mathrm{p}$ for trend $=0.03$ ); the negative association between possible Alzheimer's disease and control possibilities at work approached statistical significance $(\mathrm{p}$ for trend $=0.10)$. Working under high perceived risks for error was also associated with the diagnosis of dementia in general ( $p$ for trend $=0.001$ ) as well as with possible Alzheimer's disease ( $p$ for 
Table 3 Comparison of dementia cases with population controls and with ambulatory controls, separately

\begin{tabular}{|c|c|c|c|c|c|c|c|c|c|c|}
\hline \multirow[b]{2}{*}{ Variable } & \multirow[b]{2}{*}{ C } & \multirow[b]{2}{*}{$\%$} & \multicolumn{4}{|c|}{$\begin{array}{l}\text { Dementia cases }(n=195) \vee \text { population } \\
\text { controls }(n=122)\end{array}$} & \multicolumn{4}{|c|}{$\begin{array}{l}\text { Dementia cases }(n=195) \text { v ambulatory } \\
\text { controls }(n=107)\end{array}$} \\
\hline & & & $\mathbf{R}$ & & $\begin{array}{l}\text { Adj. } \\
\text { ORt }\end{array}$ & $95 \% \mathrm{Cl}$ & $\mathbf{R}$ & $\%$ & $\begin{array}{l}\text { Adj. } \\
\text { ORt }\end{array}$ & $95 \% \mathrm{Cl}$ \\
\hline \multicolumn{11}{|c|}{$\begin{array}{l}\text { Challenge at work (time weighted mean score on a } \\
\text { composite scale 1-5) }\end{array}$} \\
\hline$\leqslant 3.5$ & 64 & 32.8 & 27 & 32.8 & 1.0 & - & 25 & 23.4 & 1.0 & - \\
\hline$>3.5,<4.5$ & 98 & 50.3 & 77 & 63.1 & 0.5 & 0.2 to 1.0 & 65 & 60.7 & 0.4 & 0.2 to 1.0 \\
\hline $\begin{array}{l}\geqslant 4.5 \\
\mathrm{p} \text { for trend* }\end{array}$ & - & - & - & - & - & $\bar{p}=0.03$ & - & - & - & $\bar{p}=0.1$ \\
\hline \multicolumn{11}{|c|}{$\begin{array}{l}\text { Social climate at work (time weighted mean score on a } \\
\text { composite scale 1-5) }\end{array}$} \\
\hline$\leqslant 3.5$ & 81 & 41.5 & 55 & 45.1 & 1.0 & - & 51 & 47.7 & 1.0 & - \\
\hline$>3.5,<4.5$ & 81 & 41.5 & 49 & 40.2 & 1.4 & 0.7 to 2.6 & 41 & 38.3 & 1.6 & 0.8 to 3.1 \\
\hline$\geqslant 4.5$ & - & - & - & - & - & - & - & - & - & - \\
\hline$p$ for trend* & & & & & & $p=0.47$ & & & & $p=0.24$ \\
\hline \multicolumn{11}{|c|}{$\begin{array}{l}\text { Control possibilities at work (time weighted mean score } \\
\text { on a composite scale 1-5) }\end{array}$} \\
\hline$\leqslant 3.5$ & 32 & 16.4 & 18 & 14.8 & 1.0 & - & 10 & 9.3 & 1.0 & - \\
\hline$>3.5,<4.5$ & 130 & 66.7 & 86 & 70.5 & 0.6 & 0.3 to 1.4 & 82 & 76.6 & 0.5 & 0.2 to 1.2 \\
\hline$\geqslant 4.5$ & - & - & - & - & - & - & - & - & - & - \\
\hline $\mathrm{p}$ for trend* & & & & & & $p=0.44$ & & & & $p=0.02$ \\
\hline \multicolumn{11}{|c|}{$\begin{array}{l}\text { Work load (time weighted mean score on a composite } \\
\text { scale 1-5) }\end{array}$} \\
\hline$\leqslant 2.0$ & 22 & 11.3 & 19 & 15.6 & 1.0 & - & 11 & 10.3 & 1.0 & - \\
\hline$>2.0,<3.0$ & 62 & 31.8 & 47 & 38.5 & 1.5 & 0.5 to 4.2 & 48 & 44.9 & 0.7 & 0.2 to 2.1 \\
\hline$\geqslant 3.0$ & 78 & 40.0 & 38 & 31.1 & 3.8 & 1.3 to 11.0 & 33 & 30.8 & 1.8 & 0.6 to 5.7 \\
\hline \multirow{2}{*}{\multicolumn{11}{|c|}{$\begin{array}{l}\text { Perceived risks for error at work (time weighted mean } \\
\text { score on a composite scale 1-5) }\end{array}$}} \\
\hline & & & & & & & & & & \\
\hline$\leqslant 2.0$ & 105 & 53.8 & 72 & 59.0 & 1.0 & - & 60 & 56.1 & 1.0 & - \\
\hline$>2.0,<3.0$ & 39 & 20.0 & 21 & 17.2 & 0.9 & 0.4 to 2.1 & 24 & 22.4 & 1.1 & 0.4 to 2.6 \\
\hline$\geqslant 3.0$ & 18 & 9.2 & 11 & 9.0 & 1.2 & 0.4 to 3.9 & 8 & 7.5 & 3.4 & 1.0 to 11.8 \\
\hline $\mathrm{p}$ for trend* & & & & & & $p=0.08$ & & & & $p=0.001$ \\
\hline \multicolumn{11}{|c|}{$\begin{array}{l}\text { Social demands at work (time weighted mean score on a } \\
\text { composite scale 1-5) }\end{array}$} \\
\hline$\leqslant 3.5$ & 114 & 58.5 & 72 & 59.0 & 1.0 & - & 59 & 55.1 & 1.0 & - \\
\hline$>3.5,<4.5$ & 45 & 23.1 & 27 & 22.1 & 1.6 & 0.7 to 3.3 & 29 & 27.1 & 1.0 & 0.5 to 2.1 \\
\hline$\geqslant 4.5$ & 3 & 1.5 & 5 & 4.1 & 0.4 & 0.1 to 2.6 & 4 & 3.7 & 0.3 & 0.05 to 2.4 \\
\hline$p$ for trend* & & & & & & $p=0.81$ & & & & $P=0.06$ \\
\hline \multicolumn{11}{|c|}{$\begin{array}{l}\text { Supervisor support (time weighted mean score on a } \\
\text { composite scale 1-5) }\end{array}$} \\
\hline$\leqslant 3.5$ & 33 & 16.9 & 20 & 16.4 & 1.0 & - & 15 & 14.0 & 1.0 & - \\
\hline$>3.5,<4.5$ & 125 & 64.1 & 82 & 67.2 & 0.7 & 0.3 to 1.7 & 77 & 72.0 & 0.7 & 0.3 to 1.6 \\
\hline$\geqslant 4.5$ & - & - & - & - & - & - & - & - & - & - \\
\hline $\mathrm{p}$ for trend* & & & & & & $p=0.28$ & & & & $p=0.28$ \\
\hline \multicolumn{11}{|c|}{$\begin{array}{l}\text { Working time arrangements (time weighted mean score } \\
\text { on a scale } 0-2 \text { ) }\end{array}$} \\
\hline 0 & 22 & 11.3 & 11 & 9.0 & 1.0 & - & 8 & 7.5 & 1.0 & - \\
\hline$>0,<0.5$ & 119 & 61.0 & 79 & 64.8 & 0.8 & 0.3 to 2.1 & 72 & 67.3 & 1.0 & 0.3 to 3.2 \\
\hline$\geqslant 0.5$ & 21 & 10.8 & 14 & 11.5 & 0.9 & 0.2 to 3.4 & 12 & 11.2 & 1.2 & 0.3 to 5.0 \\
\hline$p$ for trend* & & & & & & $p=0.32$ & & & & $p=0.13$ \\
\hline
\end{tabular}

C, cases; $R$, control group subjects.

In the assessment of the time weighted mean score of psychosocial exposures, probands with $<10$ years estimable worktime were excluded from analysis. *To calculate tests for trend, the exposure scores were included as continuous variables in the logistic regression model.

†Adjusted for age, region, sex, dementia in parents, education, smoking (pack-years), and psychosocial network at age 30 .

trend $=0.009)$ and with vascular dementia $\quad(p$ for trend $=0.004$ ) alone.

The relation between the social demands at work and the diagnosis of dementia of any type was of borderline statistical significance ( $\mathrm{p}$ for trend $=0.05$ ); the OR for subjects with very high social demands at work (mean score of 4.5 or more) compared with those who had low to moderate social demands at work was 0.2 (95\% CI 0.04 to 1.3$)$. When the analysis was restricted to patients with Alzheimer's disease, the social demands at work lost its statistical significance $(p$ for trend $=0.19$ ). Vascular dementia alone was significantly associated with low social demands at work ( $p$ for trend $=0.01$ ) .

The following psychosocial work factors were neither significantly associated with dementia in general nor with any specific type of dementia: social climate at work; work load; supervisor support; working time arrangements.
When the analysis was restricted to dementia cases without any cardiovascular diagnoses (not shown), moderate to high challenge at work was significantly negatively associated with dementia. Furthermore, there was a significant positive dose-response relation between the work load as well as the risks for error at work and the dementia diagnosis. In contrast to the analysis of the whole case group, restriction to the cases without cardiovascular disease led to a significant positive association between the social climate at work as well as between moderate to high social demands and dementia, but not between very high social demands and dementia.

\section{DISCUSSION}

This is the first study to examine the relation between psychosocial workplace factors and dementia. The results of this study indicate a potential protective effect of psychosocial 


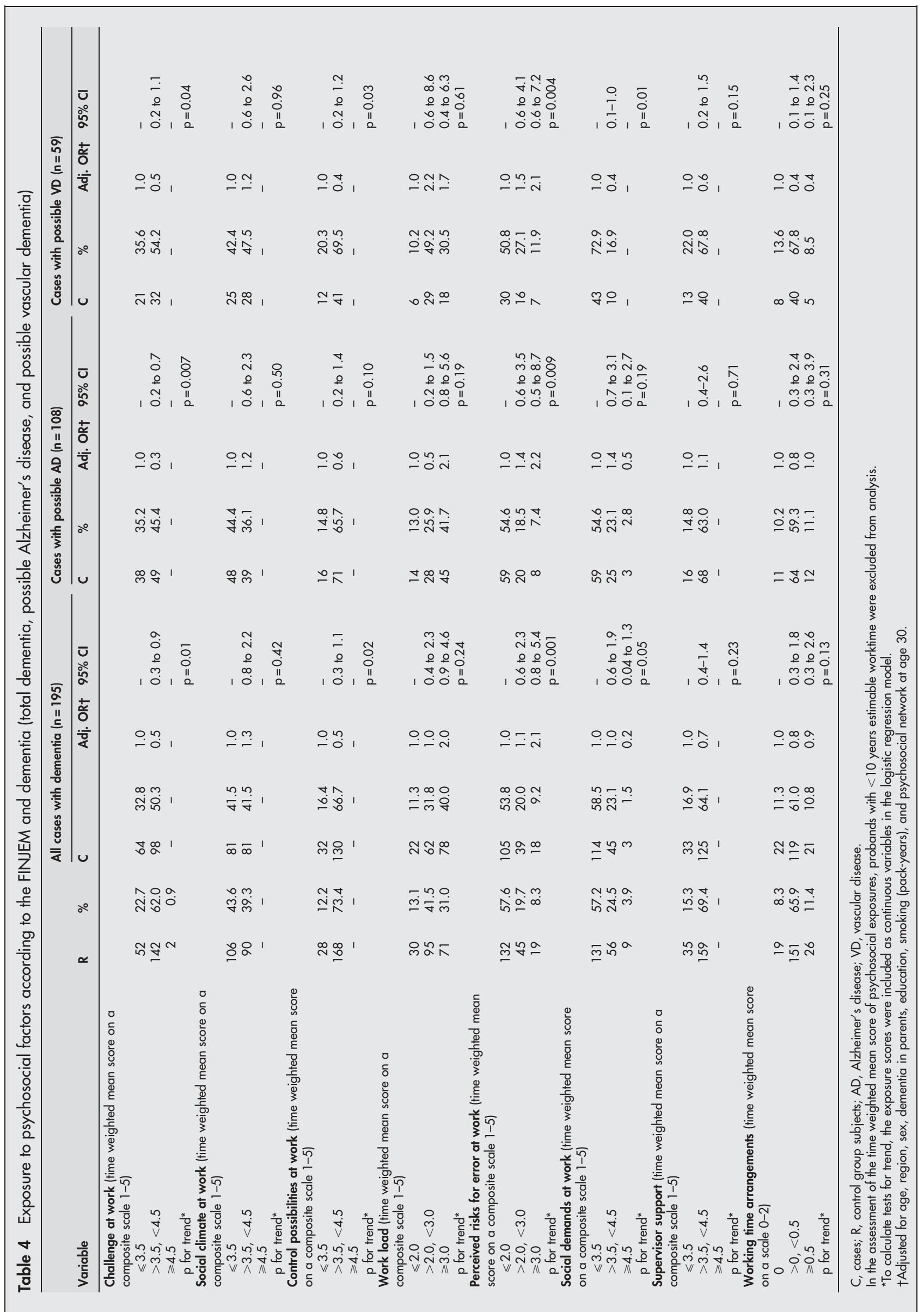


work factors-especially of a high challenge at work and high control possibilities - on dementia. In our study, odds ratios do not substantially differ between possible Alzheimer's disease and possible vascular dementia. However, as only in a minority of cases is the differential diagnosis based on neuroimaging, we cannot exclude misclassification of the type of dementia.

As in Germany almost $80 \%$ of all persons aged $70-79$ years consult a general practitioner at least once a year, ${ }^{16}$ we believe that our case recruitment procedure can be regarded as roughly population based. However, about $20 \%$ of persons aged 70-79 years either do not regularly seek any medical advice or do exclusively consult specialists. These persons are not included in our case group, but in our population control group. They might differ from persons who regularly consult a general practitioner with respect to social, psychosocial, or lifestyle factors. Moreover, control subjects that agreed to participate in our study $(61 \%)$ might differ from persons who refused to participate with respect to social status, "activity level", and state of health. By choosing ambulatory patients of general practitioners as a second control group we therefore tried to minimise potential selection bias through the case recruitment procedure and through selective participation of population controls. The response rate (90\%) was high among ambulatory control subjects; furthermore, as with the included dementia cases, these ambulatory controls regularly consulted a general practitioner. Because of the rather good agreement between the two control groups, we decided to combine both control groups in the final analysis.

Our study shows good agreement between the control subjects' and the surrogate informants' reports on job titles and duration, leading to a high concordance in psychosocial work factors classified by the FINJEM. There is no evidence for a systematic over- or underestimation of psychosocial work load on the basis of the next-of-kin job history. When only cases with partner interviews $(n=42)$ where included in the analysis, this did not fundamentally influence the results. The association between challenge at work and dementia as well as the association between control possibilities and dementia remained statistically significant. Risks for error at work and social demands at work lose their significant relation with dementia; however, numbers were small. Altogether, differential misclassification between cases and control subjects seems to be unlikely.

Nonetheless, the JEM approach can be criticised as it specifies psychosocial work factors only crudely and therefore leads to substantial non-differential misclassification. However, this potential bias would tend to lead to an underestimation of the "true" relative risks. In spite of the crude assessment, which usually leads to decreased study power, we were able to detect statistically significant risk differences.

Several previous studies have revealed a relation between blue collar work and dementia. ${ }^{17-21}$ The mechanism of this relation remains unexplained. A potential aetiological relevance to dementia of specific substances has been discussed in the literature, such as, for example, solvents ${ }^{22}$ or aluminium. ${ }^{23}$ However, up to now there has been no convincing evidence for a toxicological explanation of the relation between blue collar work and dementia. Our finding of a potential causal relation between psychosocial work factors and dementia could provide an explanation for the link between blue collar work and dementia.

Different pathological mechanisms have been discussed in the literature to explain the increased dementia risk in poorly educated people or in persons with a poor psychosocial network; ${ }^{11}$ these pathological mechanisms could also explain the potential aetiological role of poor psychosocial work factors. According to the brain reserve hypothesis, "cognitive inactivity" in blue collar occupations with monotonous tasks might decrease the synaptic reserve. Clinical symptoms might begin to appear when the number of synapses falls below a threshold level or when acute stressors exceed the brain's capacity to respond effectively. ${ }^{24}$

However, besides a causal relation between psychosocial work factors and dementia-at least in the sense of an earlier clinical manifestation of dementia-some alternative explanations for our findings must be considered. Having held challenging jobs with high social demands might just indicate high individual capacities to cope with intellectually challenging activities. According to the brain reserve hypothesis, in persons with high individual capacities the diagnosis of a clinically manifest dementia might be delayed. ${ }^{25}$

Furthermore, one has to take into consideration the potential role of cardiovascular disease as the "connecting link" between psychosocial factors and dementia. Work related stress has been reported to be associated with cardiovascular disease, ${ }^{2627}$ and cardiovascular disease is associated with increased risk of dementia. If cardiovascular disease constituted the "connecting link" between psychosocial factors and dementia, one would expect the risks to be more pronounced for vascular dementia than for Alzheimer's disease. However, in accordance with Verghese and colleagues ${ }^{28}$ our study results are similar for possible Alzheimer's disease and possible vascular dementia. To further elucidate this potential pathophysiological pathway, we have restricted the analysis to dementia cases without any cardiovascular diagnoses. Because of the small numbers $(n=46$ dementia cases without cardiovascular disease), the results of this subanalysis have to be interpreted with caution. However, the results do not support the hypothesis of cardiovascular disease constituting the connecting link between work related stress and dementia.

As a further explanation of our findings, decades before a dementia becomes clinically manifest, preclinical symptoms of dementia might influence the choice of occupation and the career. Persons with preclinical dementia symptoms might have greater difficulty in performing challenging jobs with high control possibilities. To distinguish between these alternative explanations, further pathological knowledge about the preclinical course of dementia is required.

\section{ACKNOWLEDGEMENTS}

This work was supported by a grant from the Alzheimer Forschung Initiative (AFI). We would like to thank Dr K Amadori, Dr P Geller, J Jochim, Dr U Albrecht, Dr G Amadori, Dr E Balluff, Dr H Bastian, Drs G and C Czakanski, Dr C Damm, Dr R Deml, Dr J Dorschner, Dr K Euler, Dr M Green, Dr S Gödeke-Daub, Dr G Helfrich, Dr A Jablonski, Dr A Jaschke-Eberhardt, Dr S Klimaschewski, Dr W Laforsch, Dr S Mieke, Dr U Nüsse, Dr C von Rauch, Dr C Schneider, Dr W Seher, Dr B Simon, Dr K Uhl, and Dr A Vollet for their valuable contributions to the data collection. We are grateful to Dr T Kratzsch and Dr J Peters for their participation in the consensus conference.

\section{Authors' affiliations \\ A Seidler, Institute of Occupational Medicine, Johann Wolfgang \\ Goethe-University, Frankfurt/Main, Germany \\ A Nienhaus, Institution for statutory accident insurance and prevention in the health and welfare services, Germany \\ T Bernhardt, L Frölich, Central Institute of Mental Health, Department for Gerontopsychiatry, Mannheim, Germany \\ T Kauppinen, Department of Epidemiology and Biostatistics, Finnish Institute of Occupational Health, Helsinki, Finland \\ A-L Elo, Department of Psychology, University of Jyväskylä, Finnish Institute of Occupational Health, Helsinki, Finland}

\section{REFERENCES}

1 Fratiglioni L, Wang HX, Ericsson K, et al. Influence of social network on occurrence of dementia: a community-based longitudinal study. Lancet 2000;355:1315-19. 
2 Fabrigoule C, Letenneur L, Dartigues JF, et al. Social and leisure activities and risk of dementia: a prospective longitudinal study. J Am Geriatr Soc 1995;43:485-90

3 Scarmeas N, Levy G, Tang MX, et al. Influence of leisure activity on the incidence of Alzheimer's disease. Neurology 2001;57:2236-42.

4 Wang HX, Karp A, Winblad B, et al. Late-life engagement in social and leisure activities is associated with a decreased risk of dementia: a longitudinal study from the Kungsholmen project. Am J Epidemiol 2002;155:1081-7.

5 Helmer C, Damon D, Letenneur L, et al. Marital status and risk of Alzheimer's disease. A French population-based cohort study. Neurology 1999;53:1953-8.

6 Friedland RP, Fritsch T, Smyth KA, et al. Patients with Alzheimer's disease have reduced activities in midlife compared with healthy control-group members. Proc Natl Acad Sci U S A 2001;98:3440-5.

7 Wilson RS, Mendes de Leon CF, Barnes LL, et al. Participation in cognitively stimulating activities and risk of incident Alzheimer disease. JAMA 2002;287:742-8.

8 Kondo K, Niino M, Shido K. A case-control study of Alzheimer's disease in Japan-significance of life-styles. Dementia 1994;5:314-26.

9 Kropiunigg U, Sebek K, Leonhardsberger A, et al. Psychosoziale Risikofaktoren für die Alzheimer Krankheit. Psychotherapie, Psychosomatik, medizinische Psychologie 1999;49:153-9.

10 Laurin D, Verreault R, Lindsay J, et al. Physical activity and risk of cognitive impairment and dementia in elderly persons. Arch Neurol 2001;58:498-504.

11 Seidler A, Bernhardt T, Nienhaus A, Frölich L. Association between the psychosocial network and dementia-a case-control study. J Psychiatr Res 2003;37:89-98.

12 Kauppinen T, Toikkanen J, Pukkala E. From cross-tabulations to multipurpose information systems: a new job-exposure matrix. Am J Ind Med 1998;33:409-17.

13 Dilling H, Mombur W, Schmidt MH. Internationale Klassifikation psychischer Störungen. ICD 10, Kapitel V (F). Bern, Göttingen, Toronto, Seattle: Hans Huber, 1995.

14 McKhann G, Drachman D, Folstein M, et al. Clinical diagnosis of Alzheimer's disease: report of the NINCDS-ADRDA work group under the auspices of
Department of Health and Human Services task force on Alzheimer's disease. Neurology 1984;34:939-44.

15 Wetterling T, Kanitz RD, Borgis KJ. Comparison of different diagnostic criteria for vascular dementia (ADDTC, DSM-IV, ICD-10, NINDS-AIREN). Stroke 1996;27:30-6.

16 Bergmann E, Kamtsiuris P. Inanspruchnahme medizinischer Leistungen. [Utilization of medical services available in Germany under statutory sickness insurance facilities]. Gesundheitswesen 1999;61(suppl 2):S138-44.

17 Fratiglioni L. Epidemiology of Alzheimer's disease. Issues of etiology and validity. Acta Neurol Scand Suppl 1993;145:1-70.

18 Dartigues JF, Gagnon M, Barberger-Gateau P, et al. Occupation during life and memory performance in the elderly: results of the Paquid Program. Neurology 1994;41(suppl 1):322.

19 Stern Y, Gurland B, Tatemichi TK, et al. Influence of education and occupation on the incidence of Alzheimer's disease. JAMA 1994;271:1004-10.

20 Bickel H, Cooper B. Incidence and relative risk of dementia in an urban elderly population. Psychol Med 1994;24:179-92.

21 Qiu C, Karp A, von Strauss E, et al. Lifetime principal occupation and risk of Alzheimer's disease in the Kungsholmen project. Am J Ind Med 2003:43:204-11.

22 Kukull WA, Larson EB, Bowen JD, et al. Solvent exposure as a risk factor for Alzheimer's disease: a case-control study. Am J Epidemiol 1995; 141:1059-71.

23 Martyn CN, Coggon DN, Inskip H, et al. Aluminium concentrations in drinking water and risk of Alzheimer's disease. Epidemiology 1997;8:281-6.

24 Katzman R, Kawas C. The epidemiology of dementia and Alzheimer disease. In: Terry RD, Katzman R, Bick KL, eds. Alzheimer disease. New York: Raven Press Ltd, 1994.

25 Scarmeas N, Stern Y. Cognitive reserve and lifestyle. J Clin Exp Neuropsychol 2003;25:625-33.

26 Theorell T, Karasek RA. Current issues relating to psychosocial job strain and cardiovascular disease research. J Occup Health Psychol 1996;1:9-26.

27 Peter R, Siegrist J. Psychosocial work environment and the risk of coronary heart disease. Int Arch Occup Environ Health 2000;73(suppl):S41-5.

28 Verghese J, Lipton RB, Katz MJ, et al. Leisure activities and the risk of dementia in the elderly. N Engl J Med 2003;348:2508-16. 